Review Article

\title{
A Review on Applications of Two-Dimensional Materials in Surface-Enhanced Raman Spectroscopy
}

\author{
Ming Xia (iD \\ Applied Materials Inc., Santa Clara, CA 95054, USA \\ Correspondence should be addressed to Ming Xia; xiaming@g.ucla.edu
}

Received 11 August 2017; Revised 29 October 2017; Accepted 26 November 2017; Published 1 January 2018

Academic Editor: Hakan Arslan

Copyright (C) 2018 Ming Xia. This is an open access article distributed under the Creative Commons Attribution License, which permits unrestricted use, distribution, and reproduction in any medium, provided the original work is properly cited.

\begin{abstract}
Two-dimensional (2D) materials, such as graphene and $\mathrm{MoS}_{2}$, have been attracting wide interest in surface enhancement Raman spectroscopy. This perspective gives an overview of recent developments in 2D materials' application in surface-enhanced Raman spectroscopy. This review paper focuses on the applications of using bare 2D materials and metal/2D material hybrid substrate for Raman enhancement. The Raman enhancing mechanism of 2D materials will also be discussed. The progress covered herein shows great promise for widespread adoption of 2D materials in SERS application.
\end{abstract}

\section{Introduction}

Raman is a spectroscopy technique that can provide characteristic spectral information of analytes. Due to its capability of providing fingerprints of molecule vibration, Raman spectroscopy has a wide variety of applications in chemistry, biology, and medicine [1]. However, the yield of Raman scattering is very low leading to weak Raman signals in most cases. Surface-enhanced Raman spectroscopy (SERS) makes up this deficiency via plasmon resonance or chemical enhancement. It is capable of ultrasensitive detection (singlemolecule detection) and allows for label-free detection with high degree of specificity [2-4]. There are two enhancement mechanisms accounting for SERS effect. One is electromagnetic (EM) enhancement based on plasmon resonance, which account for majority of the signal enhancement for the metallic SERS substrates. The other one is chemical enhancement, and multiple theories [5-7], such as chargetransfer mechanism, were proposed to explain it. For EM enhancement, molecules adsorbed at nanostructured metallic surface experience a large amplification of electromagnetic field due to local surface plasmon resonance leading to orders of magnitude increase in Raman yield and greatly enhanced Raman signal. To achieve high SERS enhancement factors, many efforts have been devoted to develop various metallic (mainly $\mathrm{Au}$ and $\mathrm{Ag}$ ) nanostructures to enhance the local electromagnetic field [8-12]. Two-dimensional (2D) materials, such as graphene and molybdenum disulfide $\left(\mathrm{MoS}_{2}\right)$, have unique electronic and optical properties and thus attract wide interests in their potential applications in electronic devices, sensors, and energy generation [13-18]. In addition to the above-mentioned applications, graphene and other 2D materials have also been explored to enhance Raman signals [13, 19-22]. Since the discovery of graphene's Raman enhancement capability [23], extensive researches have been done to reveal the enhancing mechanism of twodimensional materials, as well as their application in Raman enhancement either with bare 2D material substrate or with hybrid metal/2D material substrate [24-27]. This review will discuss the Raman enhancement mechanism of 2D materials and their applications in SERS.

\section{Raman Enhancement Mechanism of Two-Dimensional Materials}

Raman enhancement of two-dimensional materials is due to chemical mechanism [13, 23, 28, 29], which is different from electromagnetic enhancement mechanism of most metallic SERS substrates. Chemical enhancement mechanism of SERS has a long controversial history and is still under debate about its nature until recently. Chemical enhancement factor on metallic surface is usually low $(\sim 10)$ compared with EM 
enhancement factor, which can be as high as $10^{6}$ to $10^{11}[2,33-$ 35 ]. It has been reported that the chemical enhancement factors for pyridine on a colloidal silver surface were estimated to be 69 and 27 in the presence of $\mathrm{Cl}^{-}$and $\mathrm{ClO}_{3}{ }^{-}$ions, respectively [36]. In a broad perspective, chemical enhancement can be considered as modification of the Raman polarizability tensor of molecule upon its adsorption, which in turn enhances or quenches Raman signals of vibrational modes $[37,38]$. The observed changes in Raman spectral intensities may be explained in terms of the theory of resonance Raman. Electronic states of molecules adsorbed on metallic surface may change due to charge transfer between molecule and metal. The metal-molecule charge transfer electronic state can become resonant with the excitation wavelength so that certain Raman modes are enhanced. Before the discovery of Raman enhancement of 2D materials, investigation the chemical mechanism is hindered by its low enhancement factor and metal-adsorbate interaction. The fact that $2 \mathrm{D}$ materials have no dangling bonds in vertical direction with their atomically flat surface makes them superior platforms to investigate chemical enhancement mechanism. This section will focus on the Raman enhancement mechanism of graphene, hexagonal born nitride (h-BN), and $\mathrm{MoS}_{2}$.

Graphene is the first 2D material that was explored to enhance Raman signals of molecules [23]. Study of copper phthalocyanine ( $\mathrm{CuPc}$ )/graphene system reveals that Raman enhancement of pristine graphene is due to the groundstate charge transfer mechanism [28]. In ground-state charge transfer, adsorbates or analyte molecules do not form chemical bond with SERS substrate necessarily. Graphene is chemically inert and the charge transfer between molecules and graphene causes change in analytes' electronic distribution. Ground-state charge transfer can easily happen between graphene and molecules adsorbed on its surface because of graphene's two unique features. One is that the $\pi$ electrons are abundant on the surface of graphene. The other is that the energy band of graphene is continuous. Figure 1 shows the proposed the ground-state charge-transfer process in the graphene enhanced Raman system. In normal Raman scattered process, molecule absorbs photon energy and electrons are excited to a higher-energy level. The electrons then relax down the vibrational substructure and Raman scattered photons are emitted. The graphene electrons involvement in the Raman scattered process can enhance the electron-phonon coupling and thus induce the enhancement of the Raman signals. When a vibrational mode involves the lone pair or $\pi$ electrons, which has stronger coupling with graphene [20, 39], the vibrational mode with particular molecular orientations could have highest Raman enhancement, following the SERS selection rules. For example, CuPc molecule will be in lying-down position after annealing, which results in a molecular orientation that isoindole group is in parallel with graphene surface. $1530 \mathrm{~cm}^{-1}$ mode, which represents symmetric stretching of isoindole groups of CuPc molecules, has highest enhancement than other vibrational modes of $\mathrm{CuPc}$ after annealing [39].

To gain more insights into the relationship between distance of graphene-analyte and Raman signal, Ling and Zhang [29] investigated the Raman enhancement of mono- and

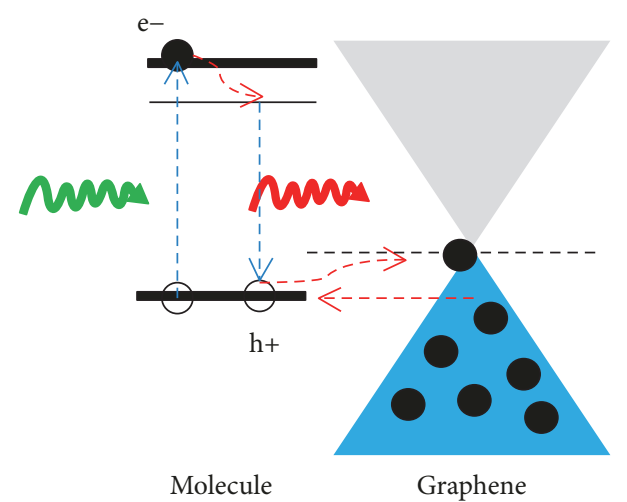

FIGURE 1: Schematic of the Raman scattered process of graphene enhanced Raman spectroscopy [28].

multilayer ordered aggregates of protoporphyrin IX (PPP) on graphene. The results indicate that most Raman enhancement is from the molecules directly contacting with graphene, which directly proves the "first-layer effect" in chemical enhancement mechanism. Functional groups close to graphene layer will be enhanced more than the group further away from the graphene, which again indicates the chemicalenhanced mechanism in this system. As seen in Figure 2, Raman peak intensity of the vinyl group or the porphyrin ring in PPP molecules will be enhanced more when PPP is on bottom position. This is because vinyl group or the porphyrin ring in that position is closer to graphene and the charge transfer is easier to happen. In addition, graphene $G$ band shifts to $1588 \mathrm{~cm}^{-1}$ from $1596 \mathrm{~cm}^{-1}$ due to charge transfer between PPP and graphene, revealing the involvement of graphene electrons in the Raman scattered process of analytes [29].

Unlike nonpolar and highly conductive graphene, h-BN is highly polar and insulating with a large band gap of $5.9 \mathrm{eV}$ [40]. CuPc molecules are found to be enhanced by h-BN substrates. One proposed Raman enhancement mechanism of h$\mathrm{BN}$ is the interface dipole interaction with analyte molecules, which causes symmetry-related perturbation in the $\mathrm{CuPc}$ molecule [13]. In addition, the Raman enhancement factor does not depend on the h-BN layer thickness, because the distribution of the intensity is uniform no matter how thick the h-BN flake is. This result suggests that $\mathrm{h}-\mathrm{BN}$ is a superior substrate regarding uniformity, when compared to graphene. Atomic layer thin $\mathrm{MoS}_{2}$ is semiconductor and has a polar bond. For $\mathrm{MoS}_{2}$, both the charge transfer and interface dipole interaction are much weaker compared with graphene and h$\mathrm{BN}$, respectively. The Raman enhancement of $\mathrm{MoS}_{2}$ is not as obvious as that of graphene and h-BN, as shown in Figure 3.

\section{Two-Dimensional Materials Alone Used as SERS Substrate}

Compared with nanostructured metallic SERS substrates, 2D materials can specifically enhance certain vibration modes of molecules due to their chemical enhancement nature. Chemisorption of a given molecule to a $2 \mathrm{D}$ material may 


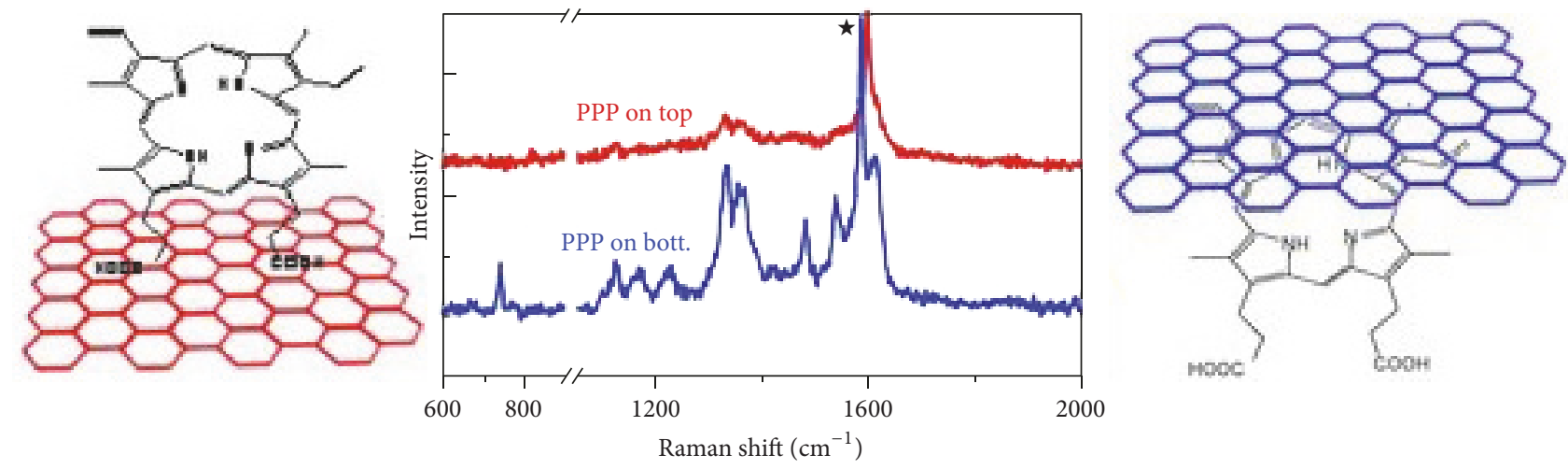

FIGURE 2: Raman spectra of PPP on the top (red line) or the bottom (blue line) of graphene. The peaks labeled with " $\star$ " are the G band from graphene [29].

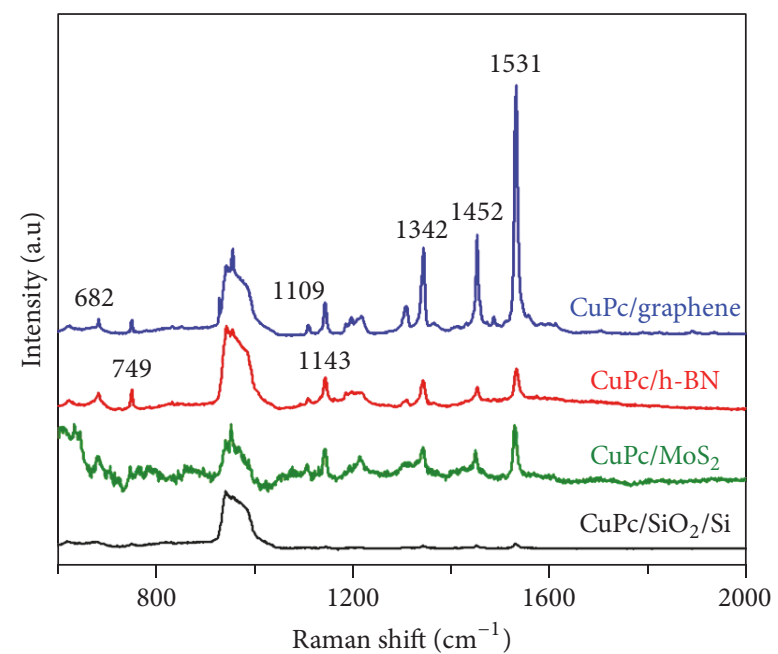

Figure 3: Raman spectra of the CuPc molecule on the blank $\mathrm{SiO}_{2} / \mathrm{Si}$ substrate, on graphene, on h-BN, and on $\mathrm{MoS}_{2}$ substrates. The numbers marked on the peaks are the peak frequencies of the Raman signals from the CuPc molecule [13].

affect the electronic states of the adsorbate and resonances could be observed with the excitation laser lines. Therefore, certain vibration modes of molecules are enhanced. In addition, 2D materials have atomically flat surface and uniform SERS enhancement factors across the whole surface, which benefits the reproducibility of SERS analysis. This section will discuss the application of bare 2D materials in SERS.

Pristine 2D materials have been widely explored to detect molecules. Pristine graphene is the first two-dimensional material used for Raman enhancement. Graphene is found to be capable of enhancing the Raman intensity of various molecules, including phthalocyanine $(\mathrm{Pc})$, rhodamine 6G (R6G), PPP, and crystal violet (CV). Graphene Raman enhancement factors are different for different vibration modes, ranging from 2 to $63[13,23]$. Vibration modes involving the lone pair or $\pi$ electrons usually have the strongest enhancement because of their stronger coupling with graphene. For instance, $1531 \mathrm{~cm}^{-1}$ peak (ring C-C stretch) of $\mathrm{CuPc}$ molecules is enhanced more by graphene than $749 \mathrm{~cm}^{-1}$ peak (in plane ring symmetric N-M stretch) [13]. Graphene substrate is also capable of suppressing fluorescence (FL) background in resonance Raman spectroscopy. Xie et al. [41] demonstrated that graphene could be used as a substrate to suppress FL background by $\sim 10^{3}$ times, which can be used to measure resonance Raman spectroscopy from fluorescent molecules such as R6G and PPP.

Besides pristine 2D materials, surface treated and functionalized 2D materials are also explored to improve SERS performance. It is demonstrated that functional group could change the doping level of graphene and thus modify the SERS signal intensity of R6G molecules [42]. Valeš et al. [42] demonstrated that fluorinated and 4-nitrophenyl functionalized graphene substrates could provide even higher enhancements for R6G molecules than pristine graphene substrate. UV/Ozone-oxidized graphene has structural disorder and defects on the graphene surface and resulted in a large chemical mechanism-based signal enhancement [43]. This oxidation along p-doping can result in very high SERS signal. Enhancement factors of UV/Ozone-oxidized graphene could reach $\sim 10^{4}$ for molecules like rhodamine $\mathrm{B}(\mathrm{RhB})$, R6G, and CV. These functionalization and surface treatments will modify the Fermi level of graphene and thus affect Raman scattering intensity of molecules on graphene through charge transfer resonance conditions [44]. Surface treated $\mathrm{MoS}_{2}$ shows enhanced Raman intensities of R6G molecules by one order of magnitude compared with pristine $\mathrm{MoS}_{2}$ flakes [45]. The introduction of defects in $\mathrm{T}-\mathrm{MoS}_{2}$ samples changes the local surface properties of $\mathrm{MoS}_{2}$ nanoflakes, such as creating the local dipoles, which give rise to the enhancement of Raman signals of R6G molecules and absorbance of the oxygen in ambient air to dope holes in $\mathrm{MoS}_{2}$, resulting in the enhanced charge transfer effect between R6G and $\mathrm{MoS}_{2}$.

In addition to single type of $2 \mathrm{D}$ materials, $2 \mathrm{D}$ heterostructure is also explored as a platform for surface-enhanced Raman scattering. Tan et al. [30] stacked $\mathrm{WSe}_{2}$ monolayer and graphene together to form a heterostructure for Raman enhancement of $\mathrm{CuPc}$ molecules. The results show that the intensity of the Raman scattering on the surface of graphene/ $\mathrm{WSe}_{2}(\mathrm{G} / \mathrm{W})$ heterostructure is much stronger compared with isolated layers, as shown in Figure 4. The enhanced Raman scattering of the CuPc molecule on the surface of the 


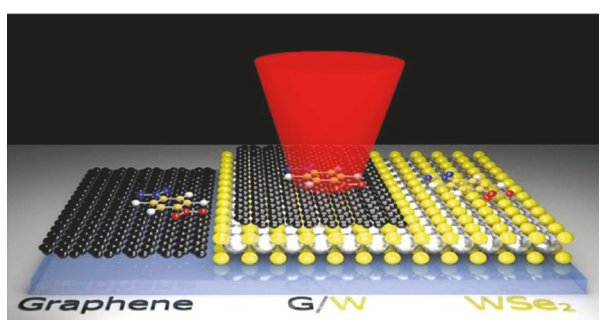

(a)

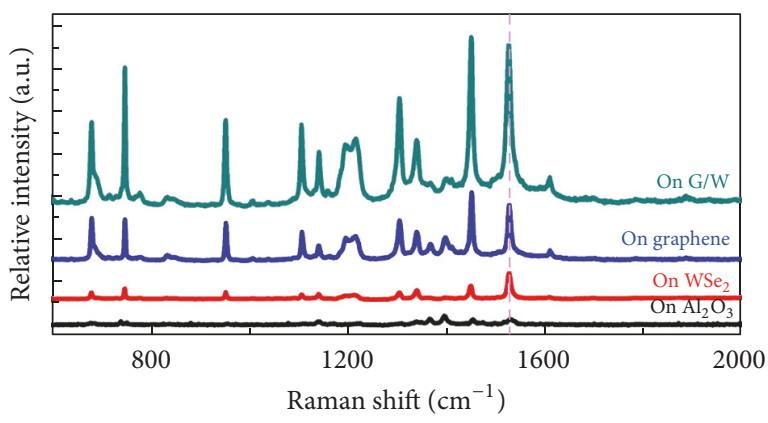

(b)

FIGURE 4: (a) Schematic illustration of the measurement procedure and prepared samples including graphene, WSe ${ }_{2}$, and G/W heterostructure. (b) Raman spectra of the CuPc molecule on the substrates of the $\mathrm{Al}_{2} \mathrm{O}_{3}$ wafer (black solid line), WSe $e_{2}$ (red solid line), graphene (blue solid line), and G/W (cyan solid line) [30].

heterostructure is due to the increasing of the charge transfer at the interface of heterostructure.

\section{Two-Dimensional Materials Combined with Metallic Nanostructures}

Traditional SERS analysis relies on metallic nanostructures that can generate strong local EM field. When combining two-dimensional materials with metallic structure, the hybrid SERS substrate can provide even higher SERS EF due to the synergic effect of EM and chemical enhancement. 2D materials, like graphene, could offer chemically inert and biocompatible surface $[46,47]$, which is favorable in biodetection. With 2D material as a shielding layer on metallic nanostructure, metal SERS platform such as Ag could be protected from oxidation and have longer shelf life, which can improve the stability and repeatability of the SERS analysis. The following discussion will mainly focus on the incorporation of graphene with various metallic SERS substrates.

Graphene/Au nanopyramid hybrid SERS platform has been explored to detect analytes like R6G and lysozyme and shows single-molecule detection capability [20]. Even for molecules with small Raman cross section, like dopamine and serotonin, graphene/Au hybrid platform can still achieve detection limit of $10^{-9} \mathrm{M}$ in simulated body fluid [20]. With graphene/Au nanopyramid hybrid SERS substrates, analytes peak hot spots and graphene peak hot spots actually coincide as seen from the Raman intensity mapping of analytes peak with that of the graphene G peak (Figure 5). These results indicate that the intrinsic Raman signal of $2 \mathrm{D}$ materials in 2D materials/metal hybrid SERS platform could be used as a marker of hotspots. Although FDTD simulation could simulate the EM field of metallic nanostructure and predict the position of hot spots, the actual hot spots of patterned nanostructure could change due to the imperfect nanofabrication. Graphene and $\mathrm{MoS}_{2}$ have been proven to be capable of overlapping on Au nanostructures and generating strong Raman signals of graphene and $\mathrm{MoS}_{2}$ [48]. With $2 \mathrm{D}$ materials' intrinsic Raman peak intensity as a SERS EF factor marker, the hot spots of the 2D materials/metal hybrid SERS platform could be located in advance and speed up the later detection of target molecules. This unique feature of hybrid platform offers an advantage for molecule detection in ultralow concentration. At extreme dilution, the spatial coincidence of an analyte molecule with the hot spots of the hybrid platform will be the key to detect molecular signal. For 2D materials used in hybrid SERS platform with patterned metallic SERS nanostructures, graphene is the ideal choice because graphene only has a few intrinsic Raman peaks and large-area high quality graphene is easily achievable. Another benefit of graphene is that its intrinsic peak intensity could be used to quantify the analytes $[20,22]$.

The fact that graphene can protect metal to be oxidized has been used in SERS substrate development [31, 49]. Ag nanostructure is known to have excellent SERS performance, but it has one major weakness that is easily to be oxidized in ambient environment. The degradation of Ag will lower the SERS performance and cause uncertainty of analysis. When single layer graphene combines with Ag nanostructure, this hybrid SERS platform could not only provide good SERS performance but also provide excellent stability in a harsh environment (sulfur) and at high temperatures $\left(300^{\circ} \mathrm{C}\right)[50]$. Liu et al. [31] combined graphene with silver SERS substrates and demonstrated that, with the shielding of graphene, the hybrid SERS-Active Substrate could achieve large-area uniformity and long-term stability. As seen in Figure 6, Ag SERS substrates with graphene protection still has high SERS enhancement performance after 6-day exposure to ambient environment, while bare Ag SERS substrate quickly loses its SERS capability by $75 \%$ even after one-day exposure. The graphene/Ag hybrid SERS platform will benefit the Raman analysis by providing longer shelf life of SERS substrate and improving SERS EF due to chemical enhancement of graphene.

Graphene could also be used as a carrier to support metal nanoparticles as SERS substrates. Ag NP/graphene or AuNP/graphene hybrid platform has been prepared and used as SERS platform, showing superior enhancement performance for various molecules [51-56]. With graphene as Ag NP carrier, nanoparticles will be uniformly distributed on graphene surface with less aggregation. Therefore, AgNP/ graphene provides more hot spots and improved SERS EF. 


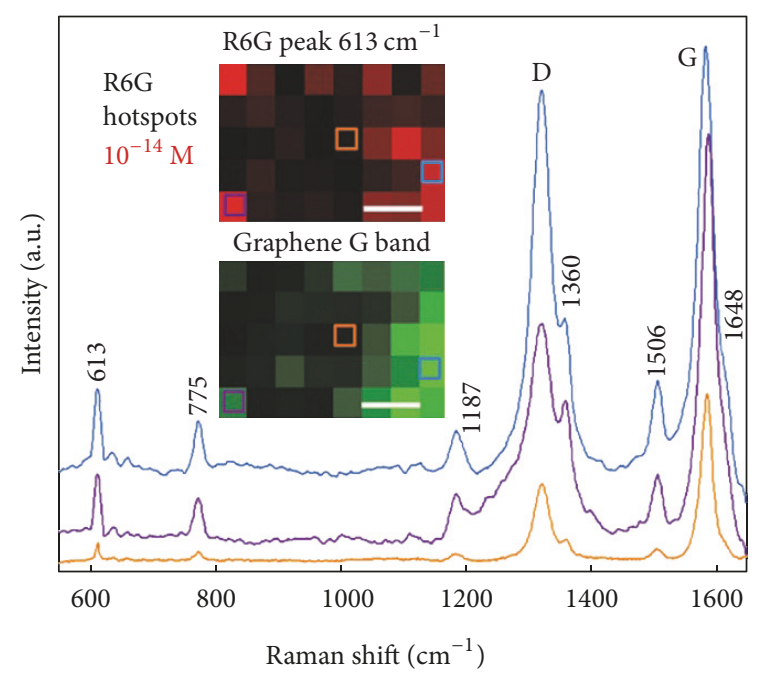

(a)

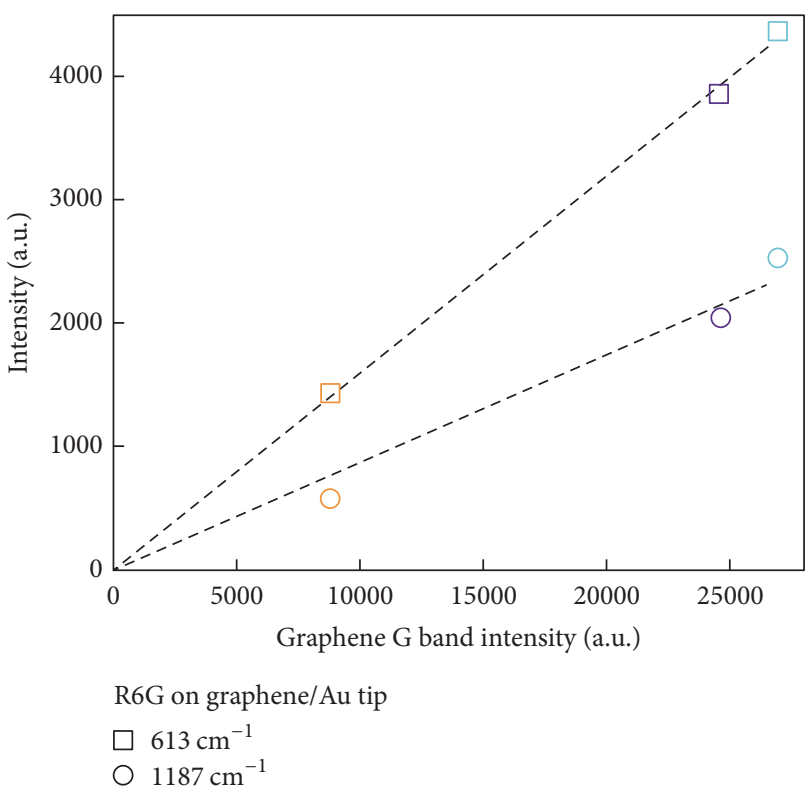

(b)

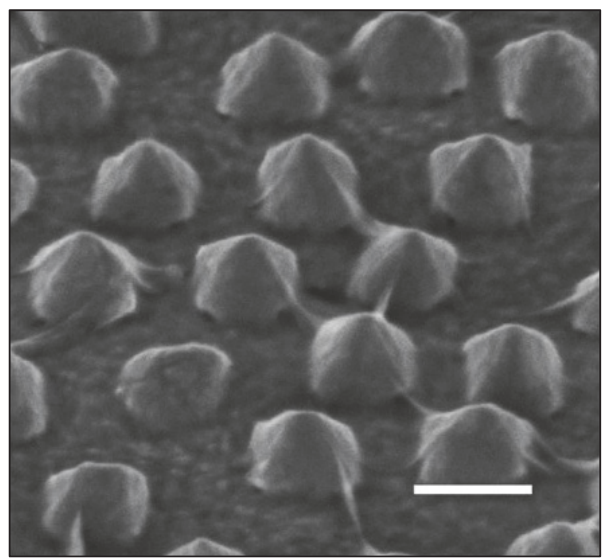

(c)

FIGURE 5: (a) SERS spectra for $10^{-14} \mathrm{M}$ R6G on graphene hybrid system taken from three spots from the inset Raman mappings in the same color squares. The inset of panel (a) is composed of Raman intensity mapping of R6G peak at $613 \mathrm{~cm}^{-1}$ (red) and Raman intensity mapping of graphene G band (green), scale bar, $2 \mu \mathrm{m}$. (b) Raman intensities of R6G peaks at $613 \mathrm{~cm}^{-1}$ and $1187 \mathrm{~cm}^{-1}$ separately as a function of graphene $\mathrm{G}$ band from the three spectra shown in panel (a). (c) SEM image of graphene/Au nanopyramid hybrid SERS platform, scale bar, $200 \mathrm{~nm}$ [20].

Employing the similar idea, Au NP or Ag NP can also be fabricated on MoS2 sheets to create hot spots and provide enhanced SERS performance [57-59]. Liang et al. [59] has reported a 3D $\mathrm{MoS}_{2}-\mathrm{NS} @ \mathrm{Ag}-\mathrm{NPs}$ nanostructure which can detect trace thiram in apple juice and local lake water with a detection limit as low as $42 \mathrm{nM}$.

Another advantage to combine graphene and metallic nanostructures it that graphene can help protect molecules from photoinduced damage, such as photobleaching [19, $32,60,61]$. The photobleaching (or photodegradation) of the Raman analytes induced by the laser is a well-known phenomenon in normal SERS experiments, especially for dye molecules. When combining graphene with metallic nanostructure, the hybrid SERS platform is more stable against photoinduced damage, but with a comparable enhancement factor. Zhao et al. [61] demonstrated that graphene underneath the organic molecules inhibited the substrateinduced fluctuations; and graphene on top of the organic molecules encapsulated and isolated them from ambient oxygen, greatly enhancing the photostability. Liu et al. [32] fabricated graphene-encapsulated metal nanoparticles for molecule detection and found out that AuNP/graphene hybrid substrate could significantly suppress photobleaching and fluorescence of phthalocyanine and R6G molecules. For instance, within the $160 \mathrm{~s}$ measurement period, the $1534 \mathrm{~cm}^{-1}$ peak intensity of CoPc molecules decreases dramatically for Au NPs, while the same peak intensity almost keeps constant for Au@Graphene, as shown in Figure 7. With 


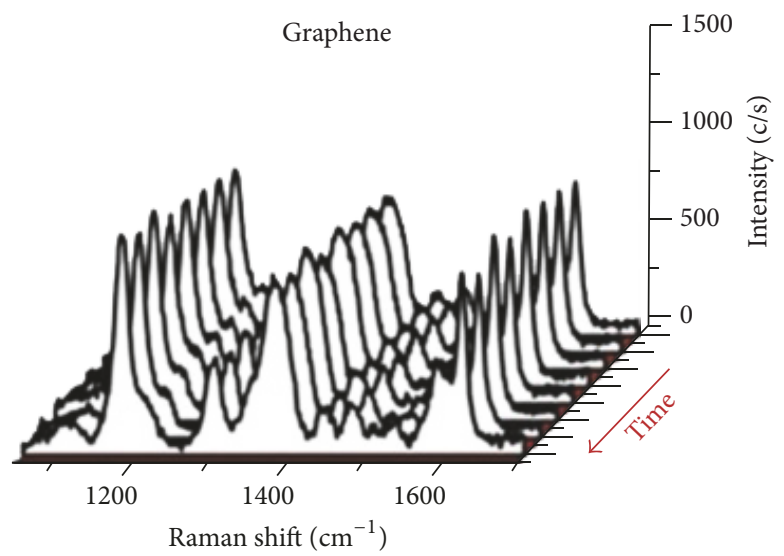

(a)

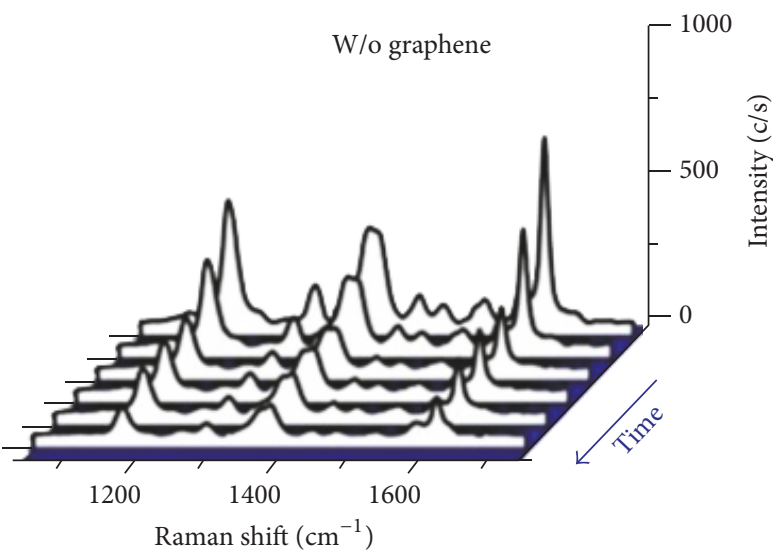

(b)

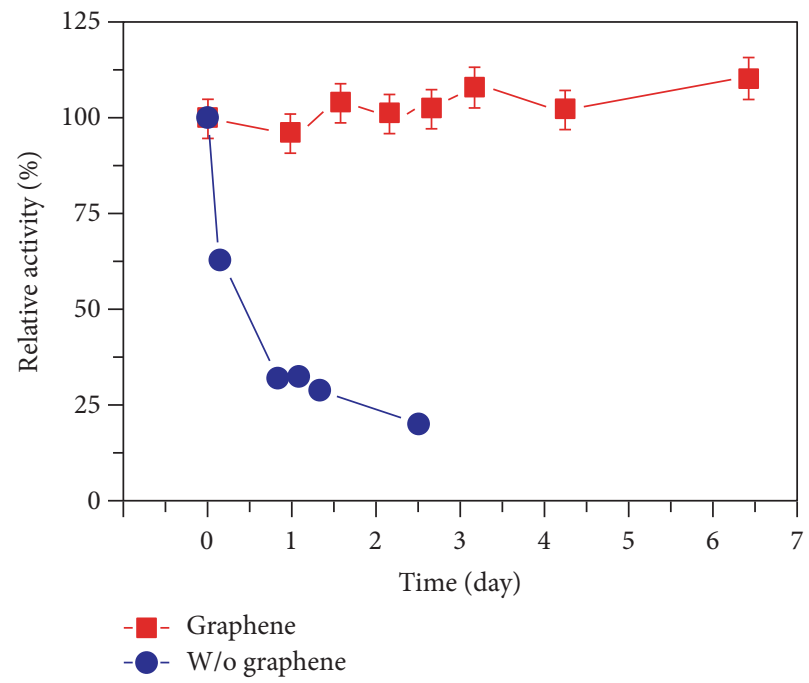

(c)

FIGURE 6: SERS spectra of CV from the SERS substrate with (a) and without (b) graphene protection at different time points. (c) The variation of the SERS intensities at $1167 \mathrm{~cm}^{-1}$ versus the time of aerobic exposure (normalized the SERS intensities before the exposure) [31].

graphene as a shielding layer, metal/graphene SERS substrates will provide long-term stability. Du et al. [60] discovered the similar phenomenon of graphene protection by simply coating graphene films on Au nanoparticles.

The benefits of adding 2D materials on metallic nanostructures lay on the following folds. First, 2D materials help further increase SERS enhancement factors due to its chemical enhancement. Although the chemical enhancement factor of $2 \mathrm{D}$ materials is not as high as metallic nanostructure, several tens' times of Raman signal enhancement could be essential when detecting molecules at single molecular level. Several times enhancement determines whether the Raman peaks can be seen or not. Second, the atomic thin film of $2 \mathrm{D}$ materials will not affect the local EM field of metallic nanostructure and can help map out the hot spots of metallic nanostructure. For instance, monolayer graphene has only $2.3 \%$ absorption of the incident laser, and its plasmon resonance frequency is the tetra $\mathrm{Hz}$ regime. Therefore, it has little effect on the EM field of underneath metallic SERS substrates. Third, a Raman mapping of graphene $\mathrm{G}$ or 2D peak over the hybrid SERS substrates could give the precise position of hot spots. With the information of hot spots location, Raman detection of target analytes could be done only at these hot spots regions and thus improve the detection efficiency especially at ultralow concentration. Finally, adding graphene as a shielding layer offers chemically inert surface and helps to reduce the fluctuation of SERS signal caused by degradation of the metallic nanostructures, photocarbonization, photobleaching, or metal-catalyzed site reactions.

\section{Conclusions}

In summary, 2D materials are unique SERS substrates, whose chemical enhancement nature differentiates them from the majority of metallic SERS substrates. SERS EF of pristine 2D materials is not comparable with that of metallic substrates. However, 2D materials have other advantages over bare metallic SERS substrates, such as chemically inert surface and uniform SERS enhancement. Adding extra atomic layer of 2D materials on surface of metallic SERS structure will help 


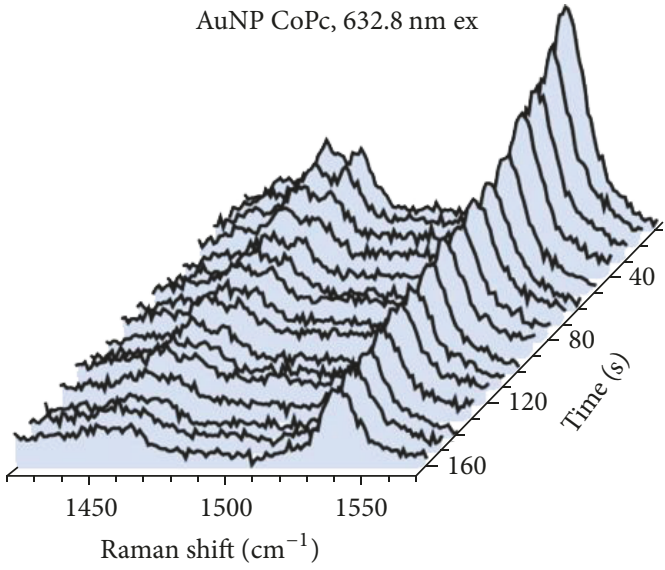

(a)

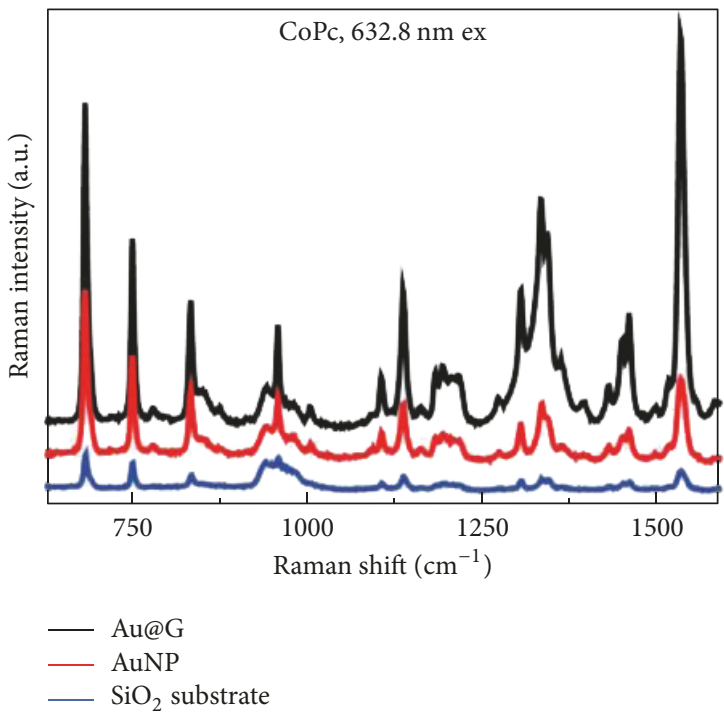

(c)

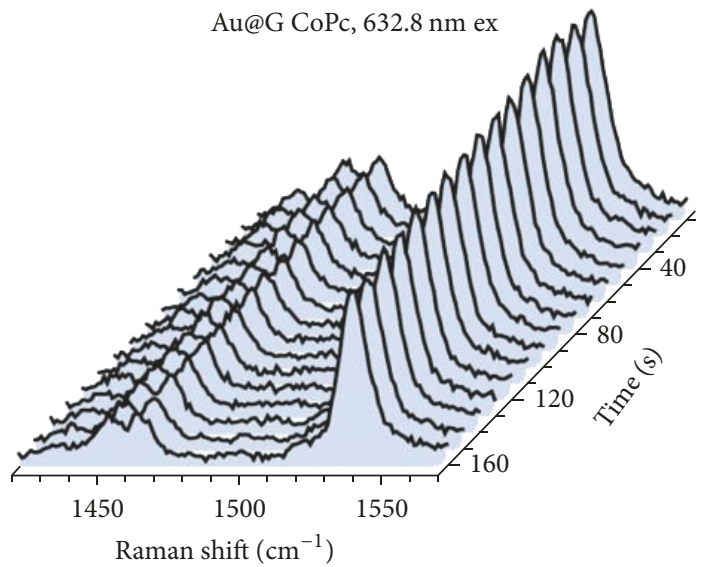

(b)

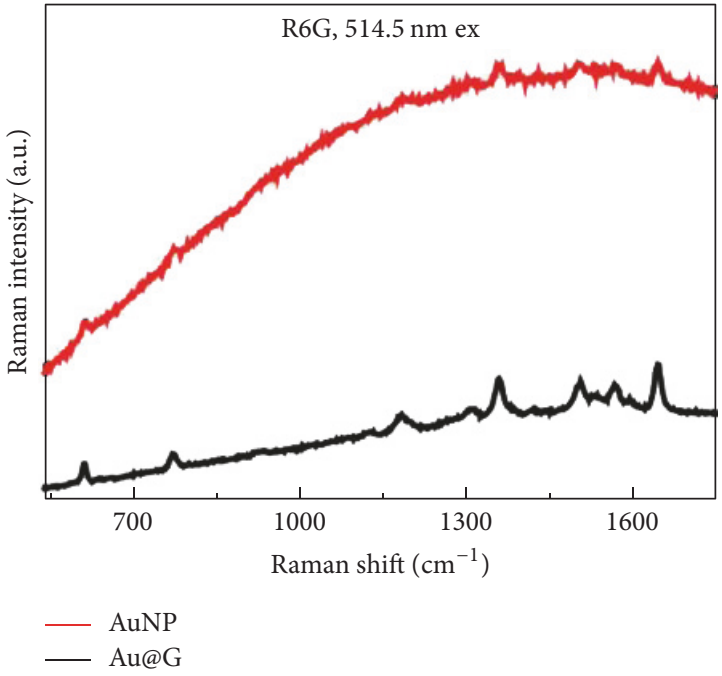

(d)

Figure 7: Stability of SERS signals of monolayer CoPc LB films on (a) Au and (b) Au@G. SERS signals of a (c) CoPc film and (d) 3 Å R6G deposited via vacuum evaporation on Au@G and Au NPs [32].

protect SERS metal form oxidation and protect molecules from photoinduced damage with even higher SERS EF due to synergetic effect of chemical and EM enhancement. Combination of 2D materials with metallic SERS structure offers a promising hybrid SERS platform, which can improve the long-term stability and repeatability of SERS analysis.

\section{Conflicts of Interest}

The author declares that there are no conflicts of interest regarding the publication of this paper.

\section{References}

[1] I. C. L. Pillai, S. Li, M. Romay et al., "Cardiac fibroblasts adopt osteogenic fates and can be targeted to attenuate pathological heart calcification," Cell Stem Cell, vol. 20, no. 2, pp. 218-232.e5, 2017.
[2] E. C. Le Ru and P. G. Etchegoin, "Single-molecule surfaceenhanced raman spectroscopy," Annual Review of Physical Chemistry, vol. 63, pp. 65-87, 2012.

[3] S.-C. Luo, K. Sivashanmugan, J.-D. Liao, C.-K. Yao, and H.-C. Peng, "Nanofabricated SERS-active substrates for single-molecule to virus detection in vitro: A review," Biosensors and Bioelectronics, vol. 61, pp. 232-240, 2014.

[4] E. J. Blackie, E. C. Le Ru, and P. G. Etchegoin, "Single-molecule surface-enhanced raman spectroscopy of nonresonant molecules," Journal of the American Chemical Society, vol. 131, no. 40, pp. 14466-14472, 2009.

[5] A. Otto, J. Billmann, J. Eickmans, U. Ertürk, and C. Pettenkofer, "The "adatom model" of SERS (Surface Enhanced Raman Scattering): The present status," Surface Science, vol. 138, no. 2-3, pp. 319-338, 1984.

[6] F. J. Adrian, "Charge transfer effects in surface-enhanced Raman scattering," The Journal of Chemical Physics, vol. 77, no. 11, pp. 5302-5314, 1982. 
[7] A. M. Michaels, M. Nirmal, and L. E. Brus, "Surface enhanced Raman spectroscopy of individual rhodamine $6 \mathrm{G}$ molecules on large Ag nanocrystals," Journal of the American Chemical Society, vol. 121, no. 43, pp. 9932-9939, 1999.

[8] J.-A. Huang, Y.-Q. Zhao, X.-J. Zhang et al., "Ordered Ag/Si nanowires array: Wide-range surface-enhanced raman spectroscopy for reproducible biomolecule detection," Nano Letters, vol. 13, no. 11, pp. 5039-5045, 2013.

[9] J. F. Li, Y. F. Huang, Y. Ding et al., "Shell-isolated nanoparticleenhanced Raman spectroscopy," Nature, vol. 464, no. 7287, pp. 392-395, 2010.

[10] W. J. Cho, Y. Kim, and J. K. Kim, "Ultrahigh-density array of silver nanoclusters for SERS substrate with high sensitivity and excellent reproducibility," ACS Nano, vol. 6, no. 1, pp. 249-255, 2012.

[11] M. Xia, K. Qiao, Z. Cheng, and Y.-H. Xie, "Multiple layered metallic nanostructures for strong surface-enhanced Raman spectroscopy enhancement," Applied Physics Express, vol. 9, no. 6, Article ID 065001, 2016.

[12] M. Xia, P. Zhang, K. Qiao, Y. Bai, and Y.-H. Xie, "Coupling SPP with LSPR for Enhanced Field Confinement: A Simulation Study," The Journal of Physical Chemistry C, vol. 120, no. 1, pp. 527-533, 2016.

[13] X. Ling, W. Fang, and Y. H. Lee, "Raman enhancement effect on two-dimensional layered materials: graphene, h-BN and $\mathrm{MoS}_{2}$," Nano Letters, vol. 14, no. 6, pp. 3033-3040, 2014.

[14] W. Zhang, R. Ma, Q. Chen et al., "The electro-mechanical responses of suspended graphene ribbons for electrostatic discharge applications," Applied Physics Letters, vol. 108, no. 15, Article ID 153103, 2016.

[15] W. Zhang, Q. Chen, M. Xia et al., "TLP evaluation of ESD protection capability of graphene micro-ribbons for ICs," in Proceedings of the 11th IEEE International Conference on Advanced Semiconductor Integrated Circuits (ASICON '15), China, November 2015.

[16] Q. Chen, R. Ma, F. Lu et al., "Systematic transient characterization of graphene interconnects for on-chip ESD protection," in Proceedings of the 2016 International Reliability Physics Symposium (IRPS '16), USA, April 2016.

[17] W. Li, X. Geng, Y. Guo et al., "Reduced graphene oxide electrically contacted graphene sensor for highly sensitive nitric oxide detection," ACS Nano, vol. 5, no. 9, pp. 6955-6961, 2011.

[18] W. Wu, L. Wang, Y. Li et al., "Piezoelectricity of single-atomiclayer MoS2 for energy conversion and piezotronics," Nature, vol. 514, no. 7253, pp. 470-474, 2014.

[19] W. Xu, X. Ling, J. Xiao et al., "Surface enhanced Raman spectroscopy on a flat graphene surface," Proceedings of the National Acadamy of Sciences of the United States of America, vol. 109, no. 24, pp. 9281-9286, 2012.

[20] P. Wang, O. Liang, W. Zhang, T. Schroeder, and Y.-H. Xie, "Ultra-sensitive graphene-plasmonic hybrid platform for labelfree detection," Advanced Materials, vol. 25, no. 35, pp. 49184924, 2013.

[21] W. G. Xu, N. N. Mao, and J. Zhang, "Graphene: a platform for surface-enhanced Raman spectroscopy," Small, vol. 9, no. 8, pp. 1206-1224, 2013.

[22] P. Wang, M. Xia, O. Liang et al., "Label-Free SERS selective detection of dopamine and serotonin using graphene-au nanopyramid heterostructure," Analytical Chemistry, vol. 87, no. 20, pp. 10255-10261, 2015.
[23] X. Ling, L. Xie, Y. Fang et al., "Can graphene be used as a substrate for Raman enhancement?” Nano Letters, vol. 10, no. 2, pp. 553-561, 2010.

[24] M. Xia, P. Zhang, C. Leung, and Y.-H. Xie, "SERS optical fiber probe with plasmonic end-facet," Journal of Raman Spectroscopy, vol. 48, no. 2, pp. 211-216, 2017.

[25] Z. Yan, M. Xia, P. Wang, P. Zhang, O. Liang, and Y.-H. Xie, "Selective manipulation of molecules by electrostatic force and detection of single molecules in aqueous solution," The Journal of Physical Chemistry C, vol. 120, no. 23, pp. 12765-12772, 2016.

[26] Z. Yan, M. Xia, P. Zhang, and Y.-H. Xie, "Self-aligned trapping and detecting molecules using a plasmonic tweezer with an integrated electrostatic cell," Advanced Optical Materials, vol. 5, no. 5, Article ID 1600329, 2017.

[27] Z. Yan, Z. Liu, M. Xia, A. Efimov, and Y. Xie, "Broadband surface-enhanced coherent anti-Stokes Raman spectroscopy with high spectral resolution," Journal of Raman Spectroscopy, vol. 48, no. 7, pp. 935-942, 2017.

[28] X. Ling, L. G. Moura, M. A. Pimenta, and J. Zhang, "Chargetransfer mechanism in graphene-enhanced Raman scattering," The Journal of Physical Chemistry C, vol. 116, no. 47, pp. 2511225118, 2012.

[29] X. Ling and J. Zhang, "First-layer effect in graphene-enhanced raman scattering," Small, vol. 6, no. 18, pp. 2020-2025, 2010.

[30] Y. Tan, L. Ma, Z. Gao, M. Chen, and F. Chen, "Two-dimensional heterostructure as a platform for surface-enhanced raman scattering," Nano Letters, vol. 17, no. 4, pp. 2621-2626, 2017.

[31] X. Liu, J. Wang, Y. Wu et al., "Compact shielding of graphene monolayer leads to extraordinary SERS-active substrate with large-area uniformity and long-term stability," Scientific Reports, vol. 5, Article ID 17167, 2015.

[32] Y. Liu, Y. Hu, and J. Zhang, "Few-layer graphene-encapsulated metal nanoparticles for surface-enhanced Raman spectroscopy," The Journal of Physical Chemistry C, vol. 118, no. 17, pp. 8993-8998, 2014.

[33] K. Kneipp, Y. Wang, H. Kneipp et al., "Single molecule detection using surface-enhanced Raman scattering (SERS)," Physical Review Letters, vol. 78, no. 9, pp. 1667-1670, 1997.

[34] J. Jiang, K. Bosnick, M. Maillard, and L. Brus, Single molecule Raman spectroscopy at the junctions of large Ag nanocrystals, ACS Publications, Washington, WA, USA, 2003.

[35] H. Xu, J. Aizpurua, M. Käll, and P. Apell, "Electromagnetic contributions to single-molecule sensitivity in surface-enhanced Raman scattering," Physical Review E: Statistical Physics, Plasmas, Fluids, and Related Interdisciplinary Topics, vol. 62, no. 3 B, pp. 4318-4324, 2000.

[36] E. J. Liang and W. Kiefer, "Chemical Effect of SERS with NearInfrared Excitation," Journal of Raman Spectroscopy, vol. 27, no. 12, pp. 879-885, 1996.

[37] E. Le Ru and P. Etchegoin, Principles of Surface-Enhanced Raman Spectroscopy: and related plasmonic effects, Elsevier, 2008.

[38] M. Moskovits, "Surface-enhanced spectroscopy," Reviews of Modern Physics, vol. 57, no. 3, pp. 783-826, 1985.

[39] X. Ling, J. Wu, W. Xu, and J. Zhang, "Probing the effect of molecular orientation on the intensity of chemical enhancement using graphene-enhanced Raman spectroscopy," Small, vol. 8, no. 9, pp. 1365-1372, 2012.

[40] C. R. Dean, A. F. Young, I. Meric et al., "Boron nitride substrates for high-quality graphene electronics," Nature Nanotechnology, vol. 5, no. 10, pp. 722-726, 2010. 
[41] L. Xie, X. Ling, Y. Fang, J. Zhang, and Z. Liu, "Graphene as a substrate to suppress fluorescence in resonance raman spectroscopy," Journal of the American Chemical Society, vol. 131, no. 29, pp. 9890-9891, 2009.

[42] V. Valeš, P. Kovaříček, M. Fridrichová et al., "Enhanced Raman scattering on functionalized graphene substrates," 2D Materials, vol. 4, no. 2, p. $025087,2017$.

[43] S. Huh, J. Park, Y. S. Kim, K. S. Kim, B. H. Hong, and J.-M. Nam, "UV/ozone-oxidized large-scale graphene platform with large chemical enhancement in surface-enhanced Raman scattering," ACS Nano, vol. 5, no. 12, pp. 9799-9806, 2011.

[44] H. Xu, L. Xie, H. Zhang, and J. Zhang, "Effect of graphene Fermi level on the Raman scattering intensity of molecules on graphene," ACS Nano, vol. 5, no. 7, pp. 5338-5344, 2011.

[45] L. Sun, H. Hu, D. Zhan et al., "Plasma modified MoS2 nanoflakes for surface enhanced raman scattering," Small, vol. 10, no. 6, pp. 1090-1095, 2014.

[46] N. Li, Q. Zhang, and S. Gao, "Three-dimensional graphene foam as a biocompatible and conductive scaffold for neural stem cells," Scientific Reports, vol. 3, article 1604, 2013.

[47] Y. Liu, D. Yu, C. Zeng, Z. Miao, and L. Dai, "Biocompatible graphene oxide-based glucose biosensors," Langmuir, vol. 26, no. 9, pp. 6158-6160, 2010.

[48] M. Xia, B. Li, K. Yin et al., "Spectroscopic Signatures of AA' and AB Stacking of Chemical Vapor Deposited Bilayer MoS2," ACS Nano, vol. 9, no. 12, pp. 12246-12254, 2015.

[49] S. Chen, L. Brown, M. Levendorf et al., "Oxidation resistance of graphene-coated $\mathrm{Cu}$ and $\mathrm{Cu} / \mathrm{Ni}$ alloy," ACS Nano, vol. 5, no. 2, pp. 1321-1327, 2011.

[50] Y. Hu, P. Kumar, Y. Xuan, B. Deng, M. Qi, and G. J. Cheng, "Controlled and stabilized light-matter interaction in graphene: plasmonic film with large-scale 10-nm lithography," Advanced Optical Materials, vol. 4, no. 11, pp. 1811-1823, 2016.

[51] Z. Zhang, F. Xu, W. Yang et al., "A facile one-pot method to highquality Ag-graphene composite nanosheets for efficient surfaceenhanced Raman scattering," Chemical Communications, vol. 47, no. 22, pp. 6440-6442, 2011.

[52] G. Lu, H. Li, C. Liusman, Z. Yin, S. Wu, and H. Zhang, "Surface enhanced Raman scattering of Ag or Au nanoparticle-decorated reduced graphene oxide for detection of aromatic molecules," Chemical Science, vol. 2, no. 9, pp. 1817-1821, 2011.

[53] X. Li, J. Li, X. Zhou et al., "Silver nanoparticles protected by monolayer graphene as a stabilized substrate for surface enhanced Raman spectroscopy," Carbon, vol. 66, pp. 713-719, 2014.

[54] W. Ren, Y. Fang, and E. Wang, "A binary functional substrate for enrichment and ultrasensitive SERS spectroscopic detection of folic acid using graphene oxide/Ag nanoparticle hybrids," ACS Nano, vol. 5, no. 8, pp. 6425-6433, 2011.

[55] Y. Zhang, S. Liu, L. Wang et al., "One-pot green synthesis of Ag nanoparticles-graphene nanocomposites and their applications in SERS, $\mathrm{H}_{2} \mathrm{O}_{2}$, and glucose sensing," RSC Advances, vol. 2, no. 2, pp. 538-545, 2012.

[56] W. Fan, Y. H. Lee, S. Pedireddy, Q. Zhang, T. Liu, and X. Y. Ling, "Graphene oxide and shape-controlled silver nanoparticle hybrids for ultrasensitive single-particle surface-enhanced Raman scattering (SERS) sensing," Nanoscale, vol. 6, no. 9, pp. 4843-4851, 2014.

[57] S. Su, C. Zhang, L. Yuwen et al., "Creating SERS hot spots on MoS2 nanosheets with in situ grown gold nanoparticles," ACS Applied Materials \& Interfaces, vol. 6, no. 21, pp. 18735-18741, 2014.
[58] D. H. Suh, "Evolution of a high local strain in rolling up MoS2 sheets decorated with Ag and Au nanoparticles for surfaceenhanced Raman scattering," Nanotechnology, vol. 28, Article ID 025603, 2016.

[59] X. Liang, Y. Wang, T. You et al., "Interfacial synthesis of a threedimensional hierarchical MoS ," Nanoscale, vol. 9, no. 25, pp. 8879-8888, 2017.

[60] Y. Du, Y. Zhao, Y. Qu et al., "Enhanced light-matter interaction of graphene-gold nanoparticle hybrid films for high-performance SERS detection," Journal of Materials Chemistry C, vol. 2, no. 23, pp. 4683-4691, 2014.

[61] Y. Zhao, Y. Xie, Z. Bao, Y. H. Tsang, L. Xie, and Y. Chai, "Enhanced SERS stability of R6G molecules with monolayer graphene," The Journal of Physical Chemistry C, vol. 118, no. 22, pp. 11827-11832, 2014. 

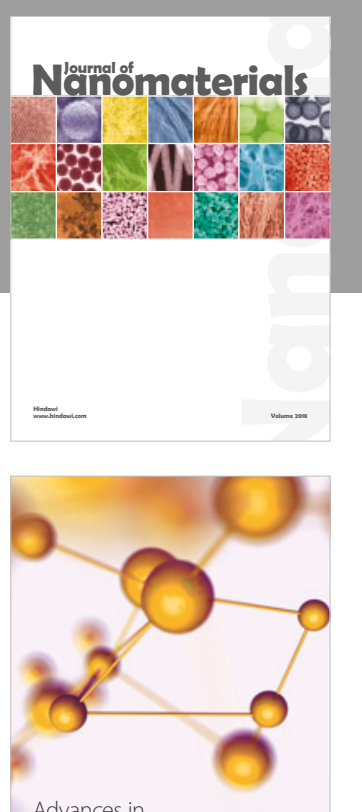

Physical Chemistry
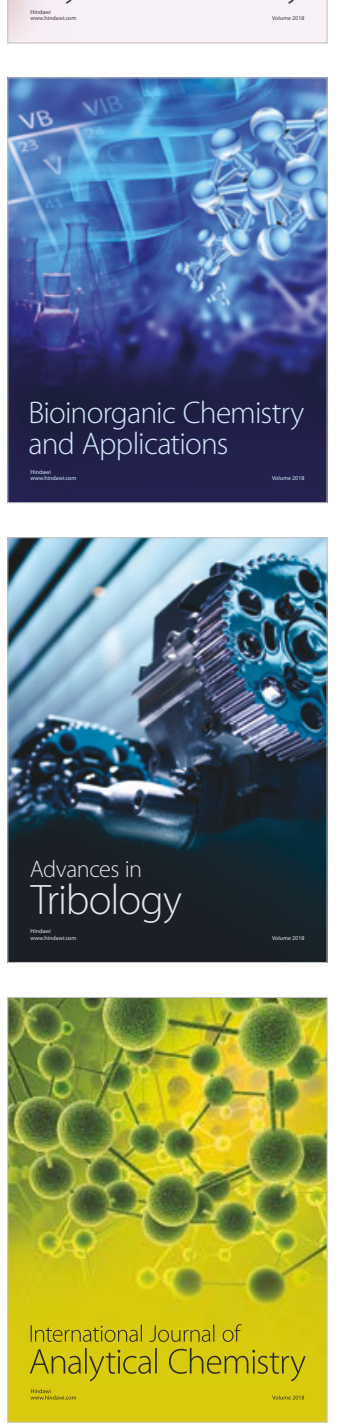

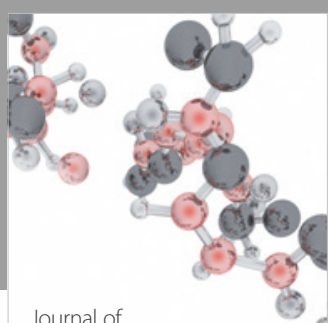

Analytical Methods

in Chemistry

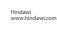

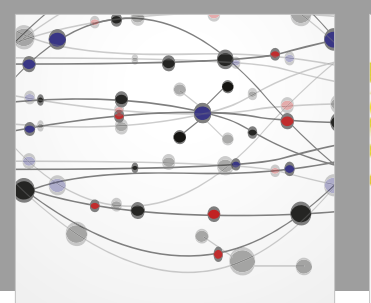

The Scientific World Journal

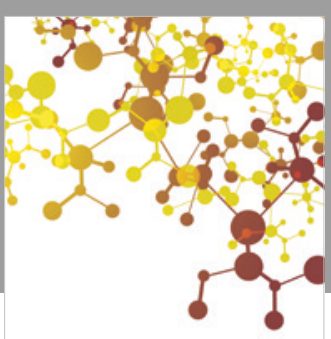

Journal of

Applied Chemistry
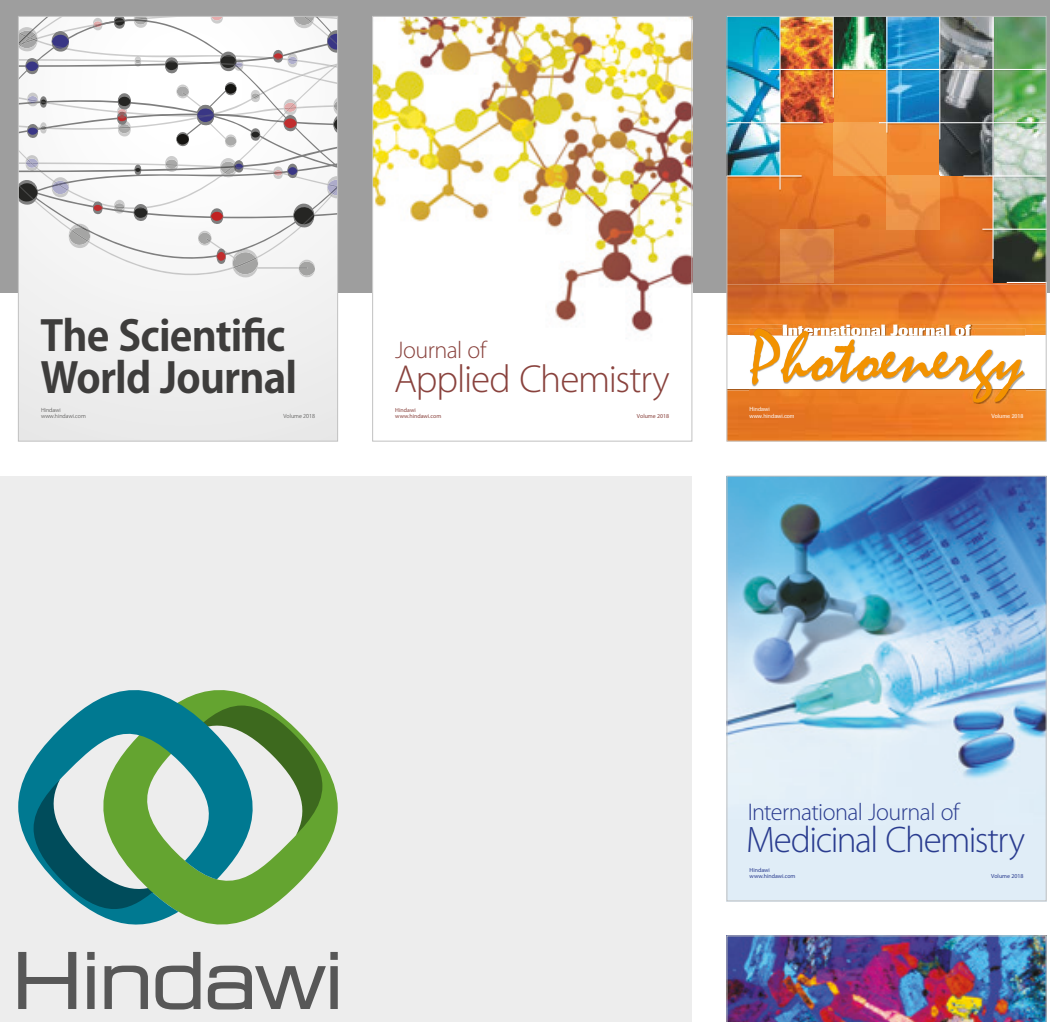

Submit your manuscripts at

www.hindawi.com
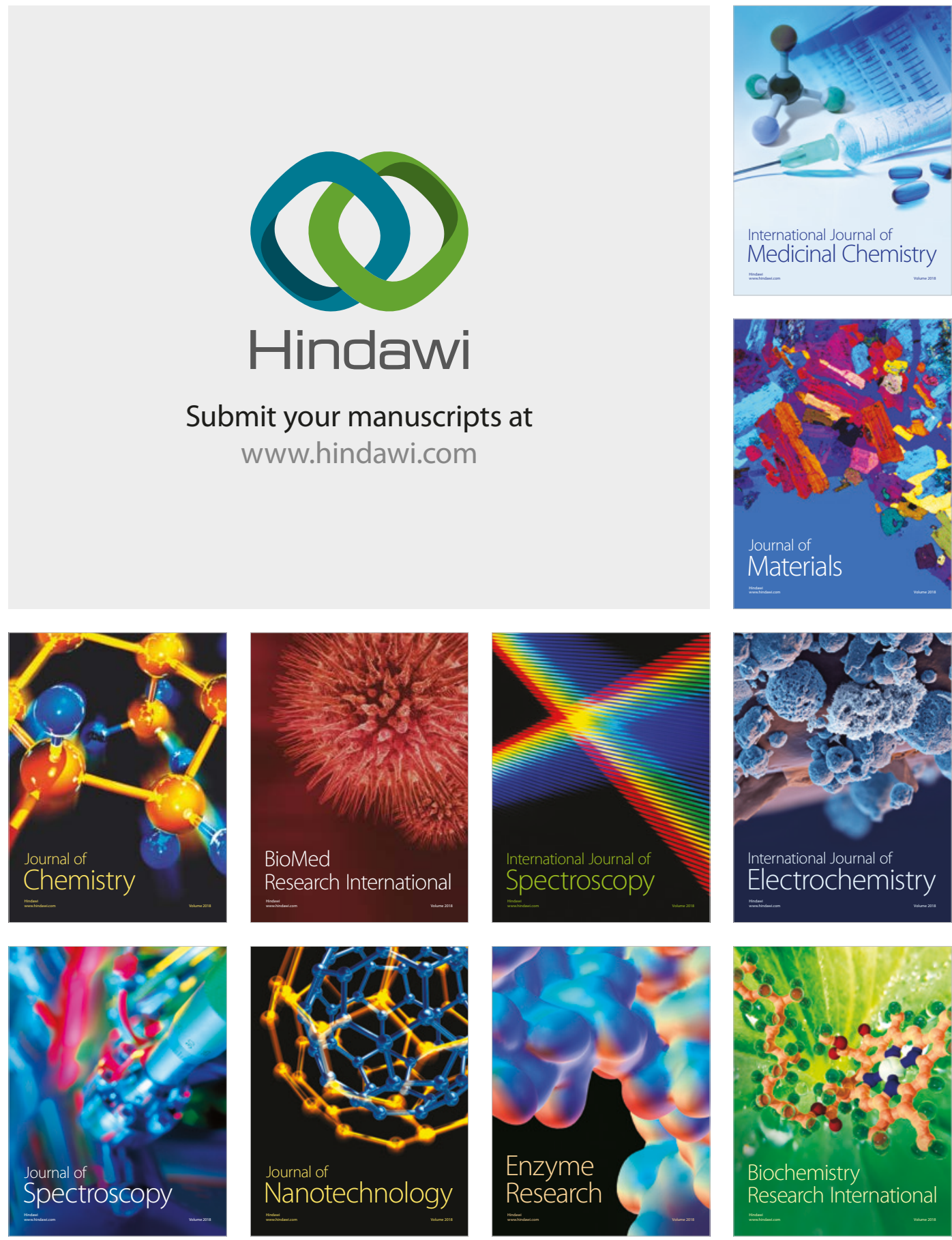
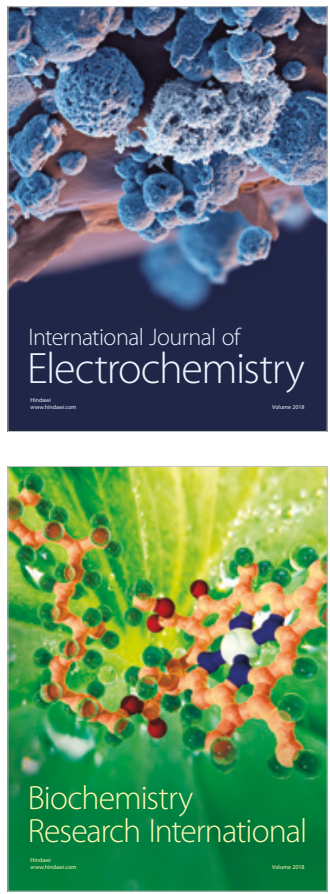